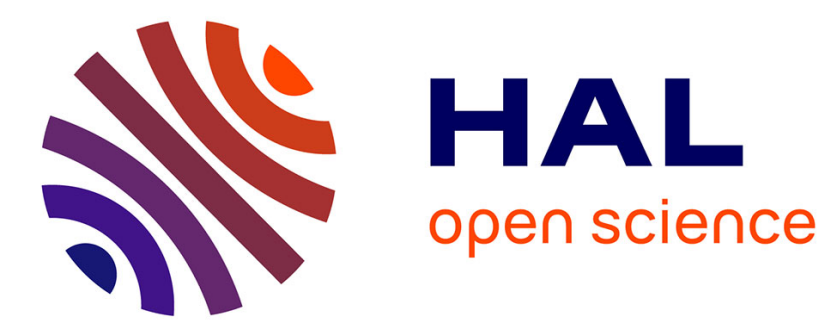

\title{
Modélisation de la dynamique de répartition de l'azote chez un jeune arbre fruitier pendant la phase de croissance exponentielle. II. Validation. Analyse de l'erreur. Simulation
}

Pascal Monestiez, Robert Habib

\section{To cite this version:}

Pascal Monestiez, Robert Habib. Modélisation de la dynamique de répartition de l'azote chez un jeune arbre fruitier pendant la phase de croissance exponentielle. II. Validation. Analyse de l'erreur. Simulation. Agronomie, 1987, 7 (7), pp.467-473. hal-00885016

\section{HAL Id: hal-00885016 https://hal.science/hal-00885016}

Submitted on 1 Jan 1987

HAL is a multi-disciplinary open access archive for the deposit and dissemination of scientific research documents, whether they are published or not. The documents may come from teaching and research institutions in France or abroad, or from public or private research centers.
L'archive ouverte pluridisciplinaire HAL, est destinée au dépôt et à la diffusion de documents scientifiques de niveau recherche, publiés ou non, émanant des établissements d'enseignement et de recherche français ou étrangers, des laboratoires publics ou privés. 


\title{
Modélisation de la dynamique de répartition de l'azote chez un jeune arbre fruitier pendant la phase de croissance exponentielle. II. Valida- tion. Analyse de l'erreur. Simulation
}

Pascal MONESTIEZ \& Robert HABIB $\left({ }^{*}\right)$

I.N.R.A., Station de Biométrie,

(*) Station d'Agronomie, Centre de Recherches d'Avignon, Domaine St-Paul, F 84140 Montfavet

RÉSUMÉ

Un modèle de transfert de l'azote endogène chez de jeunes arbres fruitiers a été proposé par les auteurs dans un article précédent (HABIB \& MONESTIEZ, 1987). Des simulations numériques sont confrontées à des données d'expériences indépendantes de celles ayant servi à la mise au point du modèle. Cette confrontation permet de considérer que le modèle est globalement validé. Cependant, l'analyse des résultats a mis en évidence certains défauts de modélisation qui sont discutés. Par ailleurs, les résidus « mesure-modèle » sont analysés. En particulier, il est démontré que les masses totales d'azote des organes végétaux peuvent être prévues avec une bonne précision ; par contre, il n'est pas possible de diminuer l'erreur résiduelle concernant les teneurs en azote. A titre d'illustration, il est montré comment ce type de modèle pourrait être employé pour préparer un plan d'expériences. Le cas d'un essai de calendrier de fertilisation est rapidement commenté.

Mots clés additionnels : Rosaceae, Prunus persica, pêcher, source, puits, réserves azotées. growth stage. II. Validation. Error analysis. Simulation.

\begin{abstract}
A model of nitrogen partitioning in young fruit trees was presented in earlier work. Numerical simulations of the model have now been compared to experimental data. These data are independent of those being used to model and fit the parameters. Simulations and experiments were in quite good agreement and the model is considered as being validated. Nevertheless some failings of the model are pointed out and discussed. The residual error " experimental data - simulated data » is analysed. The total amount of nitrogen in the vegetative parts could be predicted with good precision, but the residual error of the nitrogen content could not be lowered. An example is shown how this kind of model could be used to prepare an experimental design. An experiment on the time of application of nitrogen fertiliser is briefly discussed.
\end{abstract}

Additional key words : Rosaceae, Prunus persica, peach, source, sink, nitrogen reserve.

\section{INTRODUCTION}

Dans un article précédent (HABIB \& MONESTIEZ, 1987), les auteurs ont proposé un modèle de transfert de l'azote endogène chez un jeune arbre fruitier. L'arbre y est analysé comme un système ouvert représenté par un ensemble de compartiments anatomiques (racines, tronc, rameaux et feuilles) dont les variables descriptives sont en évolution dynamique. C'est l'organisation de ces compartiments et l'utilisation d'une équation simple régissant les débits d'azote entre organes végétaux qui ont permis de rendre compte de la dynamique des transferts de $\mathrm{N}$. L'équation de débit comprend 2 termes principaux qui traduisent la disponibilité de l'azote dans l'organe source (teneur en $\mathrm{N}$ ) et l'intensité de la demande du ou des organes puits (accroissement en matière sèche). Le 
modèle de répartition de l'azote comprend 3 paramètres qui ont été ajustés à des valeurs expérimentales.

L'expérimentation a été décrite et discutée par ailleurs (HABIB, 1987).

De jeunes scions de pêchers élevés en solution nutritive ont été soumis à 3 régimes de nutrition nitrique que l'on notera ici T1, T2, T3. Sur la base de la semaine ( 7 jours), ces traitements avaient été définis comme suit :

- Traitement dit « T1» : apport du nitrate lors du renouvellement de solution (durée du contact nitrateracines : 7 jours).

- Traitement dit « T2 2 : apport du nitrate 2 jours après le renouvellement de solution (durée du contact nitrate-racines : 5 jours).

- Traitement dit « T3» : apport du nitrate 4 jours après le renouvellement de solution (durée du contact nitrate-racines : 3 jours). Ces apports ont été réalisés à masse constante de 55 mmoles de nitrate.

Dans certains cas, deux semaines séparaient deux renouvellements de solution. Les durées de contact nitrate-racines ont alors été adaptées sur la base de 14 jours. Les 2 régimes de nutrition nitrique notés T2 et T3 étaient donc limitants quant à l'approvisionnement en nitrate.

Pendant la période de croissance exponentielle des matières sèches, 2 à 4 arbres par traitement ont été prélevés à différents pas de temps, fractionnés en racines, tronc, rameaux et feuilles, et analysés pour leurs matières sèches, MS (g), et leurs teneurs en $\mathrm{N}$ (p. 100 MS). L'évolution des masses d'azote de chaque organe au cours du temps a été obtenue par calcul. La limitation de la fourniture nitrique ne s'est pas traduite par une modification significative des croissances en MS. Par contre, les teneurs en $\mathrm{N}$ et les masses d'azote total des organes végétatifs ont été modifiées par les traitements. L'alimentation nitrique n'était donc pas limitante du point de vue de la croissance en MS mais induisait seulement des différences dans la consommation et le stockage " de luxe » de l'azote.

Ce sont les résultats relatifs au régime de fourniture nitrique non limitant (T1) qui ont servi à l'ajustement des paramètres du modèle de transfert de l'azote. L'objet principal du présent travail est de confronter des simulations du modèle à des conditions expérimentales indépendantes de celles ayant servi à l'ajustement des paramètres. Ce sont les résultats des 2 traitements limitants en $\mathrm{NO}_{3}$ (T2 et T3) qui seront utilisés pour cette validation. Par ailleurs, les composantes de l'erreur résiduelle « Mesure-Modèle » seront analysées et confrontées aux hypothèses de modélisation.

\section{VALIDATION}

Les traitements $\mathrm{T} 2$ et $\mathrm{T} 3$ ne sont différenciés que par le calendrier de fourniture du nitrate (HABIB, 1987). Les simulations des expérimentations correspondantes ont été réalisées en ne modifiant, par rapport au modèle ajusté (HABIB \& MONESTIEZ, 1987), que les entrées du modèle relatives aux conditions expérimentales. C'est-à-dire que les croissances en matière sèche et les équations de débit d'absorption racinaire de $\mathrm{NO}_{3}^{-}$et de transfert d'azote entre organes sont identiques, dans leurs formes et les valeurs des paramètres, à celles déterminées lors de la phase de mise au point et d'ajustement du modèle. Nous faisons là l'hypothèse que les rôles (sources-puits) des différents compartiments anatomiques de l'arbre ne sont pas changés, ainsi d'ailleurs que leurs intensités, puisque nous utilisons les mêmes valeurs numériques pour les paramètres du modèle ; cette hypothèse est acceptable tant que l'azote n'est pas facteur limitant de la croissance.

\section{A. Analyse globale des simulations}

Sur les figures $1 \mathrm{a}$ et $1 \mathrm{~b}$, relativement aux masses et teneurs en $N$, est reporté l'ensemble des couples mesures-modèle. Les valeurs « modèle » ont été obte-
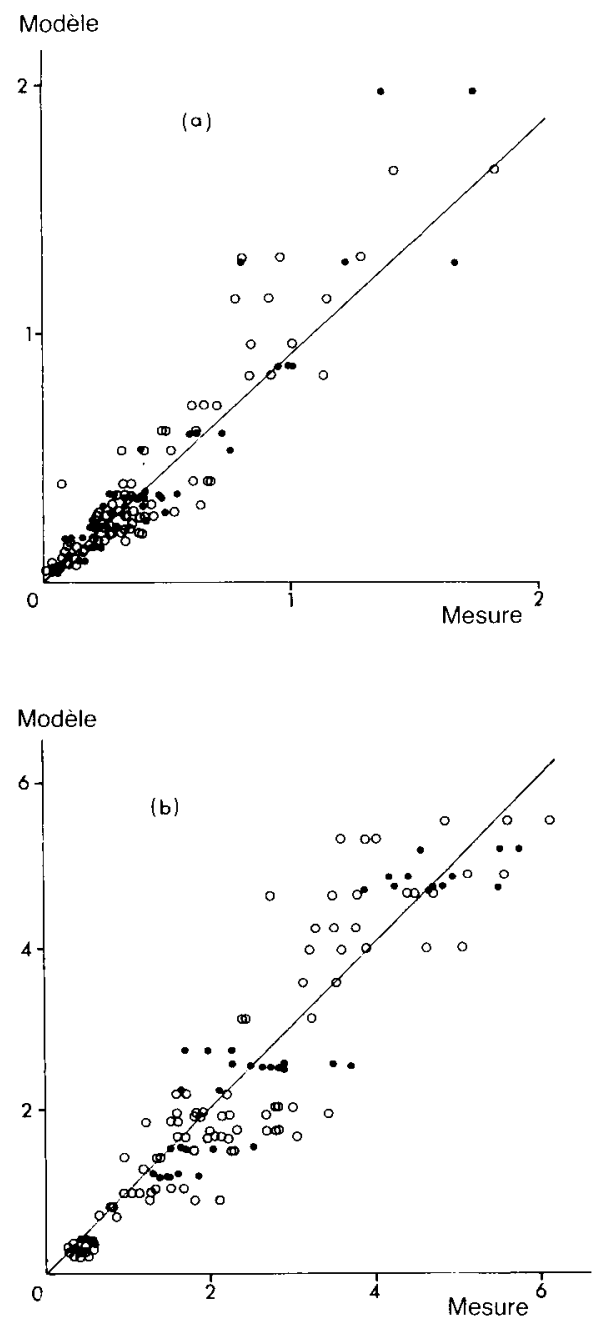

Figure 1

Comparaison globale mesure-modele

(a) Masse de $N$ (g)

(b) Teneur en $N$ (p. $100 \mathrm{MS})$

- Points issus d'ajustement (traitement TI)

Points issus de simulation (traitement $T 2$ et T3)

la première bissectrice est figurée.

Overall comparison of modelled and experimental data

(a) Amounts of nitrogen (g)

(b) Nitrogen content (dm basis)

- Modelled data from fitting

Modelled data from simulation

the first bisecting line is represented. 
nues soit après ajustement des paramètres (traitement T1 : HABIB \& MONESTIEZ, 1987), soit après simulation, stricto sensu, des expérimentations T2 et T3. Nous parlons dans ce cas de simulation car seules les entrées du modèle relatives aux conditions expérimentales (calendrier de fertilisation en particulier) ont été modifiées par rapport au modèle ajusté.

Dans les deux cas (fig. 1a et b), on n'observe pas de ségrégation des nuages de points selon qu'ils résultent d'un ajustement ou d'une simulation stricto sensu. On peut donc en conclure que l'utilisation du modèle pour simuler des expérimentations n'ayant pas été utilisées pour sa mise au point n'introduit pas de biais. Ce biais s'il existait pourrait traduire, par exemple, un manque de généralité des hypothèses de modélisation.

On peut noter cependant une sur-estimation du modèle, après ajustement, comme après simulation, pour les plus fortes masses de $\mathrm{N}$, supérieures à $1 \mathrm{~g}$ (fig. 1a), mais elle ne se retrouve pas au niveau des teneurs. Inversement, la variabilité des teneurs autour de la première bissectrice (fig. 1b) apparaît comme plus importante que celle des masses d'azote.

On peut considérer que la validation globale du modèle aux conditions expérimentales envisagées (limitation de la fourniture nitrique ne se traduisant pas par une modification des croissances en MS) est acquise. Cependant l'analyse par traitement et par organe végétal permet de nuancer ce résultat et de faire ressortir certaines limitations du modèle.

\section{B. Analyse par traitement et organe}

\section{Résultats}

Les résultats des simulations sont présentés sur les figures 2 et 4 pour les masses de $\mathrm{N}$, relativement aux traitements $\mathrm{T} 2$ et $\mathrm{T} 3$. Les figures 3 et 5 , relativement aux traitements T2 et T3, montrent les courbes d'évolution des teneurs. Les points expérimentaux y sont également figurés. Le premier point des courbes simulées (valeurs initiales des simulations) est dans chacun des cas le résultat d'une moyenne de valeurs expérimentales.

L'évolution simulée des teneurs présente une allure en "dents de scie ». Lors des simulations nous imposons, conformément aux conditions expérimentales, un arrêt de l'absorption racinaire de $\mathrm{NO}_{3}^{-}$selon le calendrier de fourniture de nitrate de chacun des deux traitements T2 et T3. Pendant cet arrêt, nous avons considéré que le système continuait à fonctionner en particulier pour les croissances en MS des organes végétaux et la dynamique de répartition de l'azote. C'est cette sur-imposition d'un sous-système bloqué (absorption racinaire) et de sous-systèmes en fonctionnement (croissance et répartition) qui explique cette allure particulière des courbes de teneurs obtenues par simulation.

L'examen des figures 2 et 4 montre que l'évolution des masses réelles d'azote des quatre organes végétaux considérés est cohérente avec la simulation en ce qui concerne les ordres de grandeur des valeurs observées. Cependant, on peut noter que les masses d'azote du tronc sont globalement sous-estimées, surtout pendant la phase de reconstitution des réserves (fig. $2 \mathrm{~b}$ et $4 \mathrm{~b}$ : $t>67 \mathrm{j}$ ). Pour les autres organes, les formes d'évolu-
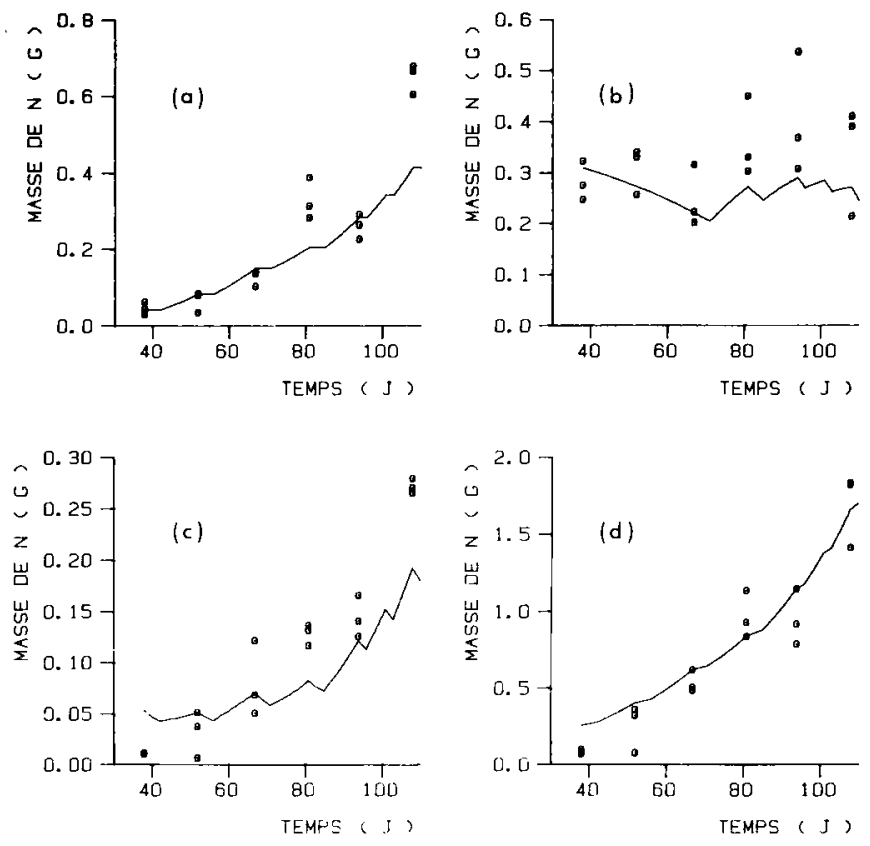

Figure 2

Evolution des masses de $N(g)$ des 4 organes pour le traitement $T 2$ : modèle et points expérimentaux. Le ler $^{\text {er }}$ point des courbes est une moyenne de valeurs expérimentales.

(a) Racines, (b) Tronc, (c) Rameaux, (d) Feuilles.

Change in amount of nitrogen in the 4 plant parts (experiment T2) : modelled and experimental data. The first point on the curves $(t=38 d)$ is taken as the average of experimental values. (a) Roots, (b) Trunk, (c) Shoots, (d) Leaves.
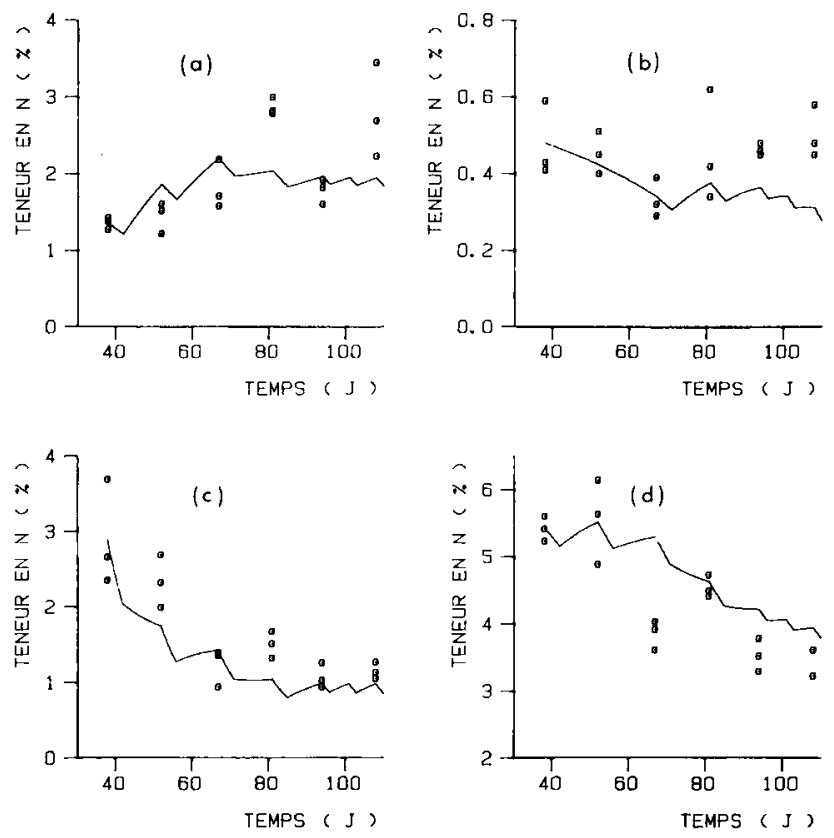

Figure 3

Evolution des teneurs en $N(p .100 \mathrm{MS})$ des 4 organes pour le traitement $T 2$ : modèle et points expérimentaux. Le ler point des courbes est une moyenne de valeurs expérimentales.

(a) Racines, (b) Tronc, (c) Rameaux, (d) Feuilles.

Change in nitrogen content (dm basis) for the 4 plant parts (experiment T2) : modelled and experimental data. The first point on the curves $(t=38 d)$ is taken as the average of experimental values. (a) Roots, (b) Trunk, (c) Shoots, (d) Leaves. 

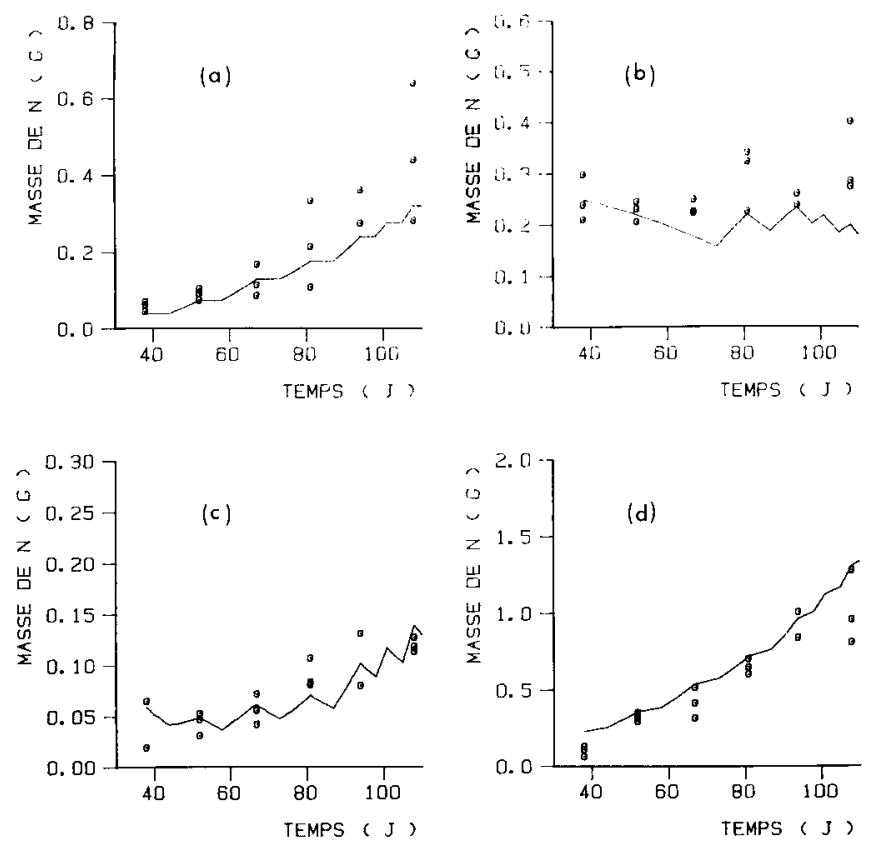

Figure 4

Evolution des masses de $N(g)$ des 4 organes pour le traitement $T 3$ : modèle et points expérimentaux. Le ler point des courbes est une moyenne de valeurs expérimentales.

(a) Racines, (b) Tronc, (c) Rameaux, (d) Feuilles.

Change in amount of nitrogen in the 4 plant parts (experiment $T 3$ ) : modelled and experimental data. The first point on the curves $(t=38 d)$ is taken as the average of experimental values. (a) Roots, (b) Trunk, (c) Shoots, (d) Leaves.
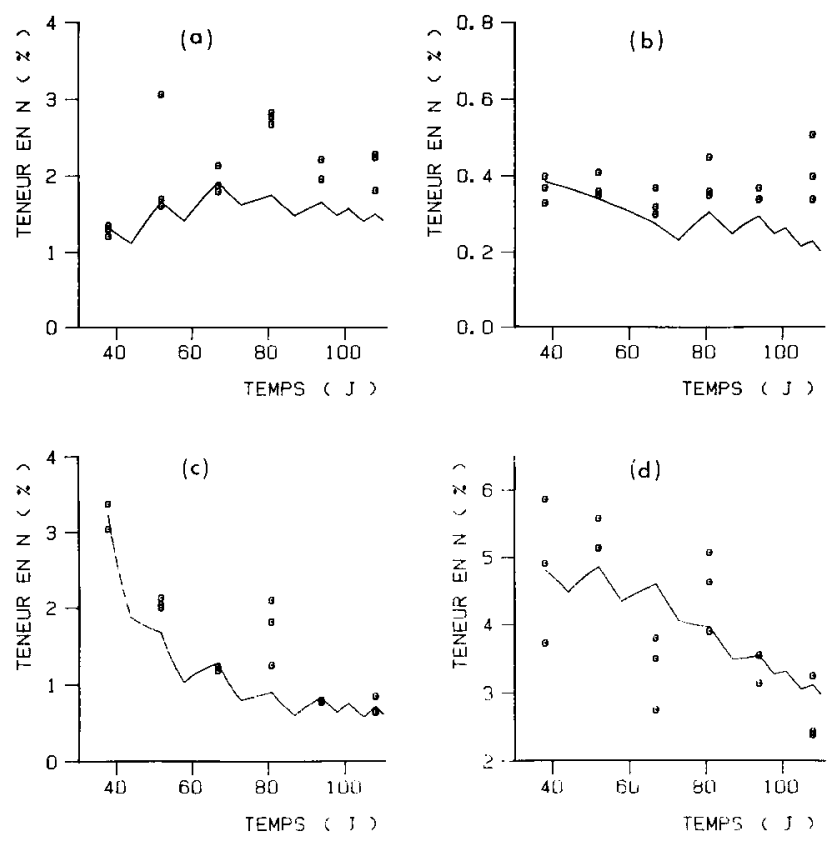

Figure 5

Evolution des teneurs en N (p. $100 \mathrm{MS})$ des 4 organes pour le traitement $T 3$ : modèle et points expérimentaux. Le $I^{\text {er }}$ point des courbes est une moyenne de valeurs expérimentales.

(a) Racines, (b) Tronc, (c) Rameaux, (d) Feuilles.

Change in nitrogen content (dm basis) for the 4 plant parts (experiment T3) : modelled and experimental data. The first point on the curves $(t=38 d)$ is taken as the average of experimental values. (a) Roots, (b) Trunk, (c) Shoots, (d) Leaves. tion des courbes sont respectées bien que l'on puisse noter une sous-estimation des valeurs simulées pour la dernière date d'observation $(t=108 \mathrm{j})$.

Concernant les teneurs (fig. 3 et 5 ) on peut également considérer que les simulations rendent compte des ordres de grandeur du phénomène : les teneurs simulées des racines ou rameaux, par exemple, ne conduisent jamais à des valeurs " impossibles ». Par ailleurs, les formes moyennes des courbes d'évolution des teneurs sont bien représentées et correspondent aux formes moyennes expérimentales communément relevées pour des organes en vieillissement (rameaux et feuilles : fig. $3 \mathrm{c}$ et $\mathrm{d}, 5 \mathrm{c}$ et $\mathrm{d}$ ). Cependant, la variabilité des résidus est nettement plus importante que celle observée pour les masses d'azote, exception faite des teneurs du tronc. Cette variabilité ne paraît pas principalement liée au caractère individuel des arbres : pour une même date, les points expérimentaux sont le plus souvent groupés. Par contre, on peut noter que, d'une date d'observation à la suivante, les teneurs semblent évoluer en « dents de scie » (augmentation puis diminution). Cette évolution cyclique n'est pas reproduite par le modèle : elle n'est ni en phase, ni du même ordre de grandeur que l'évolution en « dents de scie » liée au calendrier expérimental de fourniture de $\mathrm{NO}_{3}^{-}$aux arbres.

\section{Discussion}

On peut considérer que les hypothèses du modèle de répartition de l'azote dans l'arbre se trouvent globalement validées :

- au niveau de l'organisation topologique des compartiments végétaux,

- au niveau de l'équation régissant les débits d'azote entre organes (HABIB \& MONESTIEZ, 1987).

Cependant, les simulations ont montré un double défaut du modèle concernant la phase de reconstitution des réserves du tronc et l'évolution apparemment périodique des teneurs des autres organes. Dans le premier cas, on peut penser que la sous-estimation de l'azote du tronc est liée au modèle de croissance en MS utilisé (modèle linéaire) : l'introduction d'une croissance de type exponentiel suffirait probablement à la corriger. Dans le second cas (teneurs évoluant en « dents de scie ») il faudrait probablement modifier certaines des hypothèses de modélisation pour pouvoir en rendre compte. Il faudrait, par exemple, introduire un modèle présentant des cycles de croissance. La vérification de ce type de comportement n'apparaît pas, pour l'instant, accessible à l'expérimentation pour des arbres fruitiers.

\section{ANALYSE DES RÉSIDUS}

Le modèle, tel qu'il est construit, s'applique au fonctionnement individuel d'un arbre. L'ajustement et la validation n'ont pu être menés que sur une population d'arbres à cause de la nature même de certaines mesures. Cette population a toujours une variabilité, bien que l'on ait cherché à la rendre minimale. 
Il s'agit donc pour nous de mieux différencier au niveau des résidus ce qui provient de la variabilité inter-arbres (valeurs initiales en $\mathrm{N}$ et croissance en MS) de ce qui est réellement imputable au modèle. Notre démarche est donc de quantifier l'influence de ces différentes sources d'erreur, à l'aide d'une part, des études de sensibilité aux conditions initiales et aux commandes du système, d'autre part, en fonction du gain de variance résiduelle que permet une modélisation individuelle.

\section{A. Influence des conditions initiales}

Les valeurs initiales $(t=38 \mathrm{j})$ des masses et des teneurs en azote sont estimées pour la population à partir de quelques individus. Il est bien sûr impossible de connaître ces valeurs pour les autres, mais la variabilité observée nous permet d'étudier la sensibilité du modèle de répartition. Les courbes obtenues pour les différents organes et les deux variables (masses et teneurs) se confondent en 20 à 30 jours avec les courbes " moyennes " (modèle population) présentées sur les figures $2,3,4$ et 5 . Il ne faut donc pas voir là une cause importante d'erreur, les écarts se réduisant avec le temps, ni rechercher un moyen d'évaluer plus précisément ces valeurs initiales pour chaque arbre. Cela n'est cependant vrai que pour de jeunes scions et devra être repris dans les études ultérieures si on travaille sur les saisons de végétation suivantes.

\section{B. Influence des croissances en matière sèche}

Les croissances en MS sont les commandes principales du système de répartition de l'azote d'où l'intérêt qu'il y aurait à pouvoir ajuster une courbe par arbre et par organe. Cette procédure d'ajustement sur les MS se heurte à une difficulté essentielle. Comme on ne dispose que d'un seul point expérimental pour estimer la fonction de croissance d'un arbre individu (c'est d'ailleurs la raison pour laquelle cette procédure n'a pu être retenue dès le premier ajustement), cela nous oblige à formuler une hypothèse très forte : les courbes ont une forme établie, à un seul paramètre, et aucun contrôle de l'erreur n'est possible. Pour les racines, rameaux et feuilles, nous conservons la forme exponentielle et l'ordonnée moyenne ajustée au temps $38 \mathrm{j}$; par contre, le coefficient de l'exponentielle est ajusté pour chaque organe de chacun des arbres. Pour le tronc, c'est surtout la masse initiale qui fluctue ; nous ajustons donc en translatant verticalement la courbe «population ». Pour chaque arbre, on introduit dans le modèle complet ces nouvelles courbes de croissance MS. On obtient les réponses en MN, masse d'azote, et TN, teneur en N, que l'on appellera courbes modélisées corrigées. Certaines de ces nouvelles courbes sont représentées sur la figure 6 .

On constate, comme on pouvait logiquement s'y attendre, une grande sensibilité des masses d'azote. Les débits entre organes et le débit d'azote entrant dans le système sont en effet pilotés respectivement par les accroissements de MS et par la MS racinaire. Plus surprenante est la sensibilité comparable observée au niveau des teneurs. L'analyse des raisons en est
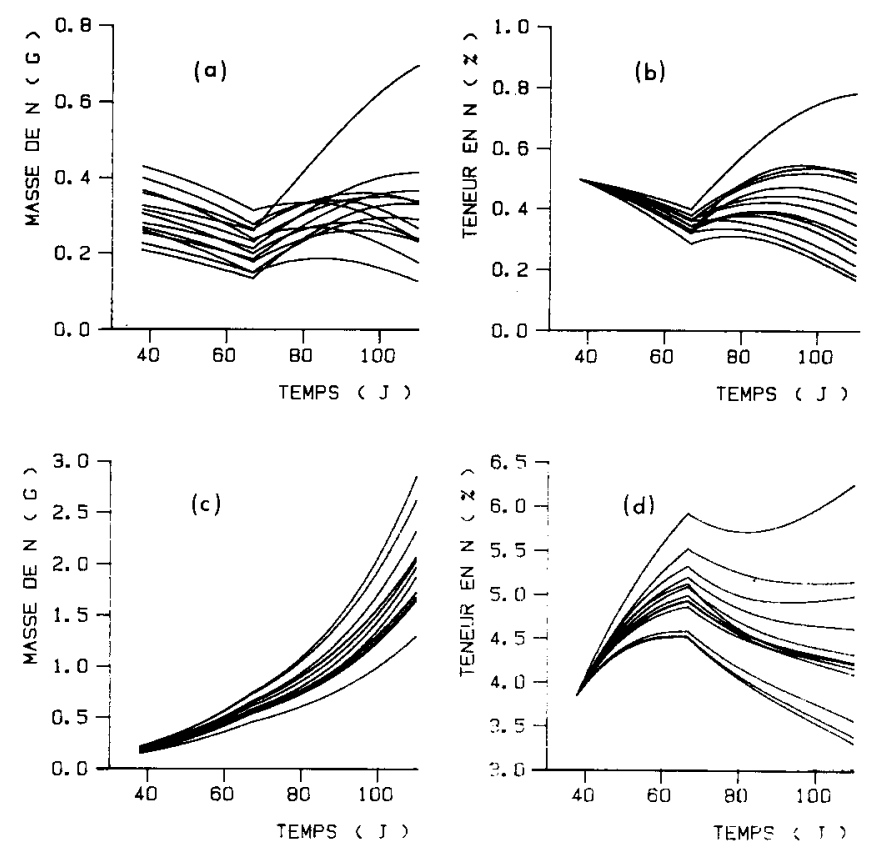

Figure 6

Sensibilité du modèle de répartition de l'azote aux cinétiques de croissance en matière sèche.

(a) et (b) Evolution en masse et teneur en $N$ du tronc. (c) et (d) Evolution en masse et teneur en $N$ des feuilles.

Sensivity of the nitrogen partitioning model to increase in dry matter.

(a) and (b) change in nitrogen amount and content in the trunk (c) and (d) change in nitrogen amount and content in the leaves.

plus complexe et les rapports de vitesses de croissance entre organes doivent en être la cause principale. Nous retiendrons donc essentiellement que des croissances différenciées d'organes entre eux et entre arbres peuvent induire des variations importantes sur les masses d'azote stockées mais aussi sur les teneurs. Le système ne tend pas à compenser ces différences induites par la croissance contrairement au cas précédent.

Dans un deuxième temps, il est intéressant de vérifier si ces nouvelles courbes modélisées corrigées ne se trouvent pas plus proches des points expérimentaux en masse et en teneur. Les nouveaux résidus sont donc comparés aux précédents en utilisant l'erreur quadratique moyenne calculée par :

$$
\sqrt{\frac{1}{n} \sum_{i} r_{i}^{2}}
$$

$r_{i}$ étant le ième résidu mesure « $\mathrm{i}$ »-modèle. L'utilisation de la variance des résidus fausserait les comparaisons car leur moyenne n'est pas exactement nulle. Les résultats sont donnés dans le tableau 1.

La correction effectuée apparaît comme efficace pour les masses d'azote $\mathrm{MN}$ et même très bonne pour la masse d'azote des feuilles. Pour les teneurs, il n'y a amélioration que pour les feuilles mais, par contre, une nette dégradation pour les racines et les rameaux. L'étude de sensibilité à la commande matière sèche nous laissait pourtant espérer qu'une correction de ce type póuvait aussi bien améliorer les résultats sur les masses que sur les teneurs en azote. Il nous faut donc approfondir nos raisonnements pour mieux comprendre ces résultats hétérogènes. 


\section{TABLEAU 1}

Correction de l'erreur résiduelle liée à l'utilisation de courbes de croissance en matière sèche individuelles pour chaque arbre.

Correction of residual error by use of dry matter increase curves fitted to each tree.

\begin{tabular}{lcccc}
\hline & $\begin{array}{c}\text { Erreur quadratique moyenne } \\
\text { Masse de } \mathrm{N} \\
(\mathrm{g})\end{array}$ & $\begin{array}{c}\text { (3 traitements confondus) } \\
\text { Teneur en N } \\
(\% \mathrm{MS})\end{array}$ \\
\cline { 3 - 5 } & Résidus & $\begin{array}{c}\text { Résidus } \\
\text { corrigés }\end{array}$ & Résidus & $\begin{array}{c}\text { Résidus } \\
\text { corrigés }\end{array}$ \\
\hline Racines & 0,114 & 0,089 & 0,66 & 0,91 \\
Tronc & 0,084 & 0,082 & 0,11 & 0,11 \\
Rameaux & 0,040 & 0,035 & 0,43 & 0,57 \\
Feuilles & 0,216 & 0,125 & 0,72 & 0,63 \\
Global & 0,130 & 0,089 & 0,53 & 0,62 \\
\hline \hline
\end{tabular}

Une analyse de ces corrections peut être aisément menée dans ce cas. Tout d'abord, il ne faut pas oublier que la masse d'azote total expérimentale n'est pas mesurée directement mais est le produit de deux mesures :

\section{MN exp $=$ TNmes $\times$ MSmes}

où $\mathrm{MS}$ est la matière sèche $(\mathrm{g}), \mathrm{MN}$, la masse de $\mathrm{N}(\mathrm{g})$ et TN, la teneur en N (p. $100 \mathrm{MS}$ ) ; par ailleurs exp signifie «expérimental » et mes : "mesuré ».

A partir des résidus sur TN et MS (modèle non corrigé) on obtient directement une décomposition fonctionnelle du résidu $\mathrm{MN}$ valable en tout point indépendamment de la dynamique antérieure de répartition de l'azote dans l'arbre.

$$
\begin{aligned}
\text { TNmes } & =\text { TNmod }+ \text { Résidu TN } \\
\text { MSmes } & =\text { MSmod }+ \text { Résidu MS }
\end{aligned}
$$

$$
\begin{aligned}
\text { MN exp }= & \text { TNmod } \times \text { MSmod (i.e. MNmod) } \\
& + \text { TNmes } \times \text { Résidu MS (i.e. Terme 1) } \\
& + \text { Résidu TN } \times \text { MSmod (i.e. Terme 2) }
\end{aligned}
$$

où mod signifie « modélisé ".

La correction effectuée en ajustant les courbes MS est principalement liée au terme 1 et très peu au terme 2 . Le terme 1 peut être calculé directement si on connaît le résidu MS au moment de la mesure. La courbe de croissance en MS ajustée individuellement passe par le point de mesure MS et rend nul le terme 1 des résidus, même si elle est complètement fausse antérieurement.

On peut donc penser que l'hypothèse utilisée pour ajuster individuellement les courbes MS est trop forte et que les croissances en MS sont différentes de ces courbes bien qu'elles aient le point expérimental en commun.

La correction faite sur la dynamique du système jusqu'au point de mesure ne diminue pas le résidu teneur, l'augmente même dans certains cas (tabl. 1). Au niveau des masses d'azote, le terme 2 du résidu tendrait donc aussi à augmenter mais cet effet est largement compensé par la correction effectuée qui annule le terme 1 des résidus.

Cette modélisation individuelle des croissances ne peut donc pas être retenue comme correcte. Les phénomènes sont sûrement plus complexes dans le détail, et la forme exponentielle ne peut être retenue que comme $1^{\text {re }}$ approximation au niveau de la population.
Dans le cas où une estimation de la répartition de l'azote à partir des valeurs de MS serait à faire, le mieux semble donc d'utiliser le modèle "population" (meilleur pour les teneurs) et d'appliquer aux masses estimées, la correction TN mod $\times$ Résidu MS (valeur approchée du terme 1) qui est satisfaisante et non négligeable.

Aller plus loin dans la validation du modèle au niveau de l'individu, demande une chose impossible techniquement : le suivi non destructif de la matière sèche. Trouver d'autres variables liées à la MS semble possible mais ajouterait d'autres erreurs. Finalement, mieux vaut s'intéresser directement à une variable indicatrice des besoins (fonction puits) dont le suivi non destructif serait possible mais qui reste à déterminer.

\section{SIMULATION D'EXPÉRIMENTATION}

Nous voudrions montrer comment ce type de modèle pourrait être employé pour préparer un plan d'expériences. Cette utilisation doit être restreinte à la période végétative pendant laquelle le modèle a été validé, ce qui en limite, pour l'instant, l'intérêt réel agronomique. A titre d'illustration, cependant, nous traiterons un essai de calendrier de fertilisation pendant la phase de croissance exponentielle des matières sèches.

Supposons que l'on veuille comparer l'effet de différentes dates de fertilisation sur l'utilisation de l'azote par de jeunes scions fruitiers. On peut simuler un essai du même ordre afin de déterminer au préalable quels seront les organes les plus affectés ou les dates d'apport les plus efficaces. Les simulations sont réalisées en comparant 10 dates de fertilisation à un témoin ; la culture étant supposée conduite dans 50 litres de solution nutritive. Pour le " témoin ", on simule pendant 10 semaines un renouvellement hebdomadaire de la solution nutritive dont la concentration initiale en $\mathrm{N}-\mathrm{NO}_{3}^{-}$est fixée à $0,01 \mathrm{~g} \mathrm{l}^{-1}$ (témoin 1) ou à $0,005 \mathrm{~g} \mathrm{l}^{-1}$ (témoin 2). Pour l'étude du calendrier de fertilisation on simule pour chacun des 10 renouvellements de solution nutritive une augmentation de la concentration initiale en $\mathrm{N}_{-} \mathrm{NO}_{3}^{-}$à $0,1 \mathrm{~g} \mathrm{l}^{-1}$. On obtient donc 10 séries de données correspondant chacune à un apport en nitrate 10 ou 20 fois plus important que le témoin, mais disponible pendant uniquement 7 jours. Les conditions expérimentales simulées permettent de rester dans le cadre de l'hypothèse de non-limitation de la croissance en MS par la fourniture nitrique.

Les résultats sont analysés en calculant l'augmentation de la masse d'azote des organes végétaux relativement au témoin 1 ou 2 à la fin de ces 7 jours de disponibilité excédentaire. Ils sont exprimés en p. 100.

Dans le premier cas (comparaison relative au témoin 1) on n'observe pratiquement pas de différences liées au calendrier de fertilisation. L'augmentation de la masse d'azote dans les organes végétaux est de l'ordre de 1 p. 100 , et peut atteindre 4 p. 100 pour les rameaux. Dans le second cas (comparaison au témoin 2), les résultats sont reportés sur la figure 7 . Les 2 organes les plus sensibles sont les rameaux et le 


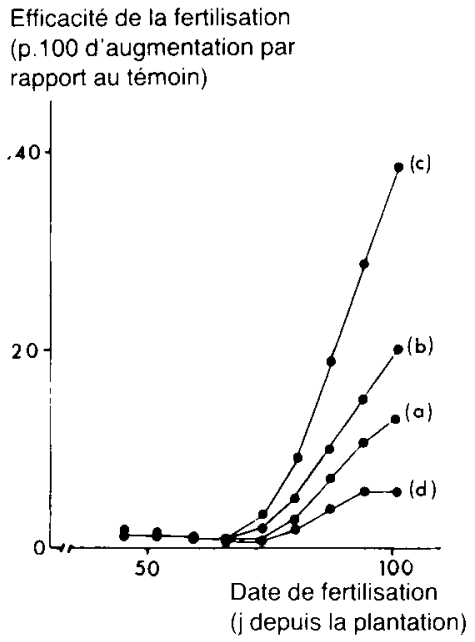

Figure 7

Simulation des courbes de réponses des 4 organes d'un arbre à un essai de calendrier de fertilisation.

(a) Racines. (b) Tronc, (c) Rameaux, (d) Feuilles.

Simulation of the sensitivity of the 4 tree parts to the time of nitrogen application.

(a) Roots, (b) Trunk, (c) Shoots, (d) Leaves.

tronc, dont l'augmentation de masse atteint, respectivement, 40 et 20 p. 100 pour la dernière date d'apport simulée. Par ailleurs, l'« efficacité » de la fertilisation augmente avec le temps, et ne devient notable que 80 jours après la plantation.

Ces résultats, s'ils devaient être utilisés pour préparer un plan d'expériences, montrent d'une part qu'il serait inutile d'envisager une fertilisation trop précoce (fig. 7: les différences ne deviennent notables que pour $t$ \# $80 \mathrm{j}$ ), et d'autre part que c'est le dosage de l'azote total des rameaux qui serait l'indicateur le plus sensible de l'efficacité des apports de $\mathrm{NO}_{3}^{-}$. Par ailleurs, il apparaît essentiel de bien contrôler le niveau du témoin de référence.

Cette simulation n'est qu'une illustration des possibilités d'utilisation du modèle. Cependant, il est intéressant de noter que l'on peut trouver dans la littérature des résultats expérimentaux allant dans le même sens, en ce qui concerne la plus grande sensibilité des rameaux à un essai de fertilisation aussi bien sur pommiers (HILl-COTTINGHAM \& COOPER, 1970) que sur pêchers (TAYLOR, 1971), pour des arbres jeunes. Par contre TAYLOR (1971) indique que, pour des pêchers adultes, les racines seraient un meilleur indicateur de l'état nutritionnel de l'arbre.

\section{CONCLUSIONS}

Le travail présenté doit être considéré comme une première approche de la modélisation de la nutrition azotée des arbres fruitiers. Pour l'instant, les hypothèses de modélisation ont été validées pendant une période végétative très courte correspondant, donc, à la phase de croissance exponentielle des matières sèches. Les expérimentations réalisées pour cette validation ont toutes été conduites en solution nutritive stricte. Cependant, nous pensons que le modèle est applicable à d'autres conditions expérimentales pour autant qu'il y ait concordance entre l'expression des besoins en azote des organes végétaux et leur croissance en MS. Le formalisme utilisé pour la modélisation (HABIB \& MONESTIEZ, 1987) permettrait, en fait, de s'affranchir de cette contrainte à condition de trouver une autre expression, que l'accroissement MS, de la fonction puits " azote " des compartiments anatomiques de l'arbre. Cependant, dans un premier temps, le plus intéressant nous paraît de tester la robustesse de l'hypothèse matière sèche à des périodes végétatives plus longues, de l'ordre de la saison de croissance.

En l'état actuel, le principal intérêt de ce type de modèle n'est pas, en fait, de prévoir la répartition des assimilats azotés dans l'arbre, mais plutôt de fournir un cadre explicatif à des expérimentations agronomiques. En effet, l'utilisation du concept "sourcepuits » conduit à relier la dynamique de distribution de l'azote dans l'arbre à l'état du système " plantemilieu ». Les conditions de sol qui peuvent influer sur l'absorption racinaire (température, fertilisation, irrigation) doivent alors être prises en compte simultanément avec le stade de développement du végétal. C'est sur cette confrontation que pourrait intervenir le cadre théorique cité précédemment. Cependant, il apparaît encore difficile de vouloir étendre la démarche de modélisation à des arbres en conditions naturelles. Les difficultés prévisibles sont de 2 ordres: trouver des moyens non destructifs de caractérisation des besoins, et applicables à différents stades de développement, et estimer l'absorption racinaire in situ.

Reçu le 7 août 1986. Accepté le 20 mars 1987.

\section{RÉFÉRENCES BIBLIOGRAPHIQUES}

Habib R., 1987. Effet de la disponibilité en nitrate sur le comportement de jeunes pêchers en culture hydroponique. Agronomie, 7 (2), 101-109.

Habib R., Monestiez P., 1987. Modélisation de la dynamique de répartition de l'azote chez un jeune arbre fruitier pendant la phase de croissance exponentielle. I. - Etablissement du modèle. Estimation des paramètres. Agronomie, 7 (6), 401-408.
Hill-Cottingham D. G., Cooper D. R., 1970. Effect of time of application of fertiliser nitrogen on the distribution and identity of the nitrogenous constituents of young apple trees. J. Sci. Food Agric., 21, 172-177.

Taylor B. K., 1971. Soluble nitrogenous fractions of tissue extracts as indices of the nitrogen status of peach trees. In Recent advances in plant nutrition, R. M. Samish Ed., Gordon and Breach, Paris, 241-249. 